\title{
ESTRESSE OCUPACIONAL RELACIONADO À ASSISTÊNCIA DE ENFERMAGEM EM TERAPIA INTENSIVA
}

\author{
OCCUPATIONAL STRESS RELATED TO NURSING \\ CARE IN INTENSIVE CARE
}

\section{ESTRÉS OCUPACIONAL RELACIONADO CON LA ATENCIÓN DE ENFERMERÍA EN CUIDADOS INTENSIVOS}

\author{
Rosana Santos Mota ${ }^{1}$ \\ Valdenir Almeida da Silva ${ }^{2}$ \\ Isadora Goncalves Brito ${ }^{3}$ \\ Ângela de Souza Barros ${ }^{4}$ \\ Olga Maria Brito dos Santos \\ Andreia Santos Mendes ${ }^{6}$ \\ Lorena de Carvalho Souza ${ }^{7}$
}

Como citar este artigo: Mota RS, Silva VA, Brito IG, Barros AS, Santos OMB, Mendes AS, et al. Estresse ocupacional relacionado à assistência de enfermagem em terapia intensiva. Rev baiana enferm. 2021;35:e38860 .

Objetivo: estimar a prevalência de estresse ocupacional entre profissionais de enfermagem que atuam em Unidade de Terapia Intensiva e identificar sua associação com variáveis sociodemográficas, profissionais e relacionadas à assistência de enfermagem. Método: estudo transversal, realizado em um hospital de ensino de Salvador, Bahia, Brasil, com 54 profissionais. Os dados foram coletados entre fevereiro e março de 2020 por meio da Escala Bianchi de Stress e analisados pelo Programa Stata. Resultados: a prevalência de estresse ocupacional em nível médio ou alto foi de 57,4\%. Maiores níveis de estresse foram associados significativamente ao menor tempo de formação ( $p$-valor=0,05), ser enfermeiro ( $p$-valor=0,00), enfrentar a morte do paciente ( $\mathrm{p}$-valor=0,01), atender aos familiares dos pacientes críticos ( $\mathrm{p}$-valor=0,00) e atender às necessidades dos familiares ( $\mathrm{p}$-valor=0,00). Conclusão: a elevada prevalência de estresse ocupacional, bem como os fatores associados identificados, foram informações essenciais para implementação de estratégias preventivas.

Descritores: Estresse Ocupacional. Cuidados de Enfermagem. Unidade de Terapia Intensiva. Equipe de Enfermagem. Enfermagem.

Objective: to estimate the prevalence of occupational stress among nursing professionals working in the Intensive Care Unit and to identify its association with sociodemographic, professional and nursing care-related variables. Method: cross-sectional study, conducted in a teaching hospital in Salvador, Babia, Brazil, with 54 professionals.

\footnotetext{
Enfermeira. Doutora em Enfermagem. Hospital Universitário Professor Edgard Santos, Universidade Federal da Bahia. Salvador, Bahia, Brasil. rosanal7santos@yahoo.com.br. http://orcid.org/0000-0002-3193-9972.

Enfermeiro. Doutor em Enfermagem. Hospital Universitário Professor Edgard Santos, Universidade Federal da Bahia. Salvador, Bahia, Brasil. http://orcid.org/00000003-1947-468X.

Bacharelanda interdisciplinar em saúde. Universidade Federal da Bahia. Salvador, Bahia, Brasil. http://orcid.org/0000-000 I-8927-8225.

Enfermeira. Especialista em Controle de Infecção Hospitalar. Hospital Universitário Professor Edgard Santos, Universidade Federal da Bahia. Salvador, Bahia, Brasil. http://orcid.org/0000-0003-4508-9056.

5 Enfermeira. Mestre em Enfermagem. Hospital Universitário Professor Edgard Santos, Universidade Federal da Bahia. Salvador, Bahia, Brasil. http://orcid.org/0000$0001-6405-2895$.

6 Enfermeira. Mestre em Enfermagem. Hospital Universitário Professor Edgard Santos, Universidade Federal da Bahia. Salvador, Bahia, Brasil. http://orcid.org/00000002-6929-395।.

Enfermeira. Hospital Universitário Professor Edgard Santos, Universidade Federal da Bahia. Salvador, Bahia, Brasil. http://orcid.org/0000-0003-1979-7032.
} 
Data were collected between February and March 2020 using the Bianchi Stress Scale and analyzed by the Stata Program. Results: the prevalence of occupational stress at medium or high level was 57.4\%. Higher stress levels were significantly associated with shorter training time ( $p$-value=0.05), being a nurse ( $p$-value=0.00), facing patient death (p-value=0.01), attending to critically ill patients' relatives $(p$-value $=0.00)$ and meeting the needs of family members (p-value=0.00). Conclusion: the high prevalence of occupational stress, as well as the associated factors identified, were essential information for the implementation of preventive strategies.

Descriptors: Occupational Stress. Nursing Care. Intensive Care Unit. Nursing Team. Nursing.

Objetivo: estimar la prevalencia del estrés ocupacional entre los profesionales de enfermería que trabajan en la Unidad de Cuidados Intensivos e identificar su asociación con variables sociodemográficas, profesionales y de cuidados de enfermería. Método: estudio transversal, realizado en un bospital de enseñanza en Salvador, Babia, Brasil, con 54 profesionales. Los datos fueron recopilados entre febrero y marzo de 2020 utilizando la Escala de Estrés de Bianchi y analizados por el Programa Stata. Resultados: la prevalencia del estrés ocupacional a nivel medio o alto fue del 57,4\%. Los niveles de estrés más altos se asociaron significativamente con un tiempo de entrenamiento más corto ( $p$-valor-0,05), ser una enfermera ( $p$-valor-0,00), enfrentar la muerte del paciente ( $p$-valor-0,01), atender a los familiares de los pacientes en estado crítico (p-value-0,00) y satisfacer las necesidades de los miembros de la familia (p-valor-0,0O). Conclusión: la alta prevalencia del estrés ocupacional, así como los factores asociados identificados, fueron información esencial para la aplicación de estrategias preventivas.

Descriptores: Estrés Ocupacional. Cuidado de Enfermería. Unidad de Cuidados Intensivos. Equipo de Enfermería. Enfermería.

\section{Introdução}

O estresse é um estado gerado pela apreensão de estímulos externos que desencadeiam excitação emocional. Tal estado contribui para o desequilíbrio da homeostasia e o disparo de uma reação de adaptação caracterizada pelo aumento de secreção de adrenalina e cortisol, provocando manifestações sistêmicas que, se persistentes, podem resultar em distúrbios fisiológicos e psicológicos $^{(1)}$. Quando associados às questões relacionadas ao trabalho, é denominado estresse ocupacional $^{(1-2)}$.

O enfrentamento do estresse ocupacional dá-se de forma individual e subjetiva. Cada pessoa pode agir de forma diferente frente às mesmas circunstâncias. A Organização Mundial de Saúde (OMS) admite que exigências relacionadas ao trabalho, pressões excessivas, conhecimentos, habilidade do trabalhador e suas reações frente às cobranças determinam a manifestação do estresse. Diante de uma situação potencialmente estressante, a capacidade da pessoa e seus recursos para enfrentá-la são confrontados. Assim, certo nível de estresse laboral pode ser considerado aceitável quando contribui para manter o profissional alerta, motivado e em condições de aprender e trabalhar. Quando tal pressão é excessiva ou difícil de controlar, há um desequilíbrio entre as exigências e pressões que o profissional enfrenta, por um lado, e seus conhecimentos e capacidades para lidar com ela, por outro, elevando, assim, os níveis de estresse laboral $^{(2)}$.

Diversos fatores contribuem para o desenvolvimento do estresse na enfermagem. Alguns deles estão intrinsecamente relacionados à profissão, como o lidar com a dor e com o sofrimento, o trabalho com pacientes em situação de enfermidade terminal e morte, as situações de urgência e aqueles relacionados à organização, como dimensionamento insuficiente de pessoal, sobrecarga de trabalho e dificuldades no relacionamento com a própria equipe de enfermagem e com outros profissionais. Como consequência, há elevação dos riscos para o aparecimento de transtornos psicológicos relacionados ao estresse, insatisfação, desmotivação, absenteísmo, trocas frequentes de postos de trabalho e abandono da profissão. Acrescenta-se também que, além das repercussões psicológicas, pode haver prejuízos à dimensão física dos trabalhadores, 
a exemplo das doenças cerebrovasculares, cardíacas e metabólicas ${ }^{(3-5)}$.

As Unidades de Terapia Intensiva (UTI) são reconhecidas por possuírem elevadas fontes de estresse. São unidades assistenciais complexas e altamente especializadas. O labor incessante associado à sobrecarga de trabalho e às condições ambientais próprias, como temperatura baixa, barulho, falta de visão externa, iluminação artificial nas 24 horas do dia e controle de acesso podem conduzir ao esgotamento emocional e levar a maior risco de estresse ${ }^{(5-7)}$.

O estresse ocupacional traz efeitos negativos tanto para o trabalhador quanto para a organização, como referido. Para a organização, tais efeitos podem ser sentidos na desorganização, na quebra de procedimentos-padrão e na queda de produtividade ${ }^{(8)}$. No que diz respeito ao estresse relacionado ao trabalho da enfermagem intensivista, há ampla documentação, produzida por diversos autores no Brasil ${ }^{(9-11)}$ e em outros países $^{(5,7)}$. O estresse e a fadiga são dois importantes fatores que contribuem para a ocorrência de erros na enfermagem, sobretudo na UTI. Profissionais fatigados cometem mais erros no julgamento clínico ou durante a administração de medicamentos e ainda falham em identificar erros cometidos por outros membros da equipe ${ }^{(7,12)}$.

O objetivo deste artigo é estimar a prevalência de estresse ocupacional entre profissionais de enfermagem que atuam em Unidade de Terapia Intensiva e identificar sua associação com variáveis sociodemográficas, profissionais e relacionadas à assistência de enfermagem.

\section{Método}

A pesquisa respeitou os princípios éticos contidos na Resolução n. 466/2012, que trata das questões éticas referentes às pesquisas que envolvem seres humanos. O projeto foi aprovado pelo Comitê de Ética em Pesquisa do Hospital de Ensino (HE), Certificado de Apresentação para Apreciação Ética (CAEE) n ${ }^{\circ}$ 09111119.4.0000.0049.

Estudo observacional, transversal e com abordagem quantitativa. A escrita do manuscrito norteou-se pelos critérios da ferramenta
Strengthening the Reportingof Observacional Studies in Epidemiology (STROBE). Foi realizado em um HE localizado na cidade de Salvador, Bahia, Brasil. A referida instituição pertence ao Sistema Único de Saúde (SUS). É uma unidade hospitalar e ambulatorial de grande porte, referência em média e alta complexidade, com 277 leitos ativos e certificada desde 2004 como hospital de ensino pelos Ministérios da Saúde e da Educação. O HE possui duas unidades de terapia intensiva adulto, compreendendo 20 leitos destinados ao cuidado de pacientes críticos. A equipe de enfermagem é composta por 40 enfermeiros e 68 técnicos em enfermagem.

Participaram do estudo 54 profissionais de enfermagem, sendo 18 enfermeiros e 36 técnicos/auxiliares de enfermagem. A proposta do estudo seria abranger todos os profissionais de enfermagem que atendessem ao critério de inclusão de atuar na UTI por mais de seis meses. No entanto, devido à pandemia do novo Coronavírus, a coleta de dados foi interrompida, tendo contemplado aproximadamente 50\% dos profissionais. Foram excluídos da pesquisa todos os colaboradores que se encontravam de férias e afastados por motivo de doença ou de licença maternidade.

A coleta de dados foi realizada entre os meses de fevereiro e março de 2020 por uma bolsista de iniciação científica, devidamente treinada pelos pesquisadores responsáveis. $\mathrm{O}$ instrumento utilizado foi a Escala Bianchi de Stress (EBS) ${ }^{(13)}$, construída e validada no Brasil em 2009, que avalia o estresse no desempenho das atividades profissionais. Trata-se de uma escala autoaplicável, contendo 51 itens do tipo Likert, com variação de 1 a 7: o valor 1 é considerado como pouco desgastante; o valor 4, como médio; e o valor 7, como altamente desgastante. O valor 0 é reservado para quando o profissional não executa a atividade abordada. Esta escala é dividida em seis domínios: (A) relacionamento com outras unidades; (B) funcionamento adequado da unidade; (C) administração de pessoal; (D) assistência prestada ao paciente; (E) coordenação das atividades da unidade; e (F) condições de trabalho ${ }^{(13)}$. 
Para avaliar o nível geral de estresse, as pontuações entre 1 e 119 correspondem a um baixo nível de estresse; de 120 a 238, médio nível de estresse; e de 239 a 357, alto nível de estresse $^{(13)}$. Foram considerados profissionais com maiores níveis de estresse os que apresentaram pontuação equivalente ao nível médio ou alto. As variáveis sociodemográficas e profissionais investigadas foram: idade, sexo, raça, estado civil, religião, categoria profissional, tempo de formação, tempo de trabalho no HE, vínculo empregatício, graduação e pós-graduação.

As variáveis relacionadas à assistência de enfermagem prestada ao paciente constituem o domínio D da EBS, motivo pelo qual foi eleito para atender o objetivo do estudo. Compõem esse domínio: admissão do paciente, exame físico, prescrição de enfermagem, avaliar as condições do paciente, atender as necessidades do paciente, atender as necessidades dos familiares, orientar o paciente para o autocuidado, orientar os familiares para o autocuidado do paciente, supervisionar o cuidado de enfermagem prestado, orientar para a alta do paciente, prestar os cuidados de enfermagem, atender as emergências na unidade, atender aos familiares dos pacientes críticos, enfrentar a morte do paciente, orientar familiares dos pacientes críticos. Estas variáveis foram categorizadas em "Sim" e "Não", referindo-se ao maior desgaste do profissional para a sua execução (pontuações que variaram entre 5 e 7 pontos), e nenhum, ao menor desgaste para o desempenho das atividades (pontuações que variaram entre 0 e 4 pontos), respectivamente.

A organização dos dados deu-se por meio do programa Microsoft Excel 2007, constituindo o banco de dados. Em seguida, foram transportados para o Programa Stata versão 12, no qual foi realizada a análise estatística. Os dados descritivos foram reportados como frequências e percentagens. Para testar associação entre as variáveis, foram utilizados os testes do Qui-quadrado e exato de Fisher. Foi calculada razão de chances (odds ratio) com respectivo intervalo de confiança de 95\%, utilizando regressão logística. Foi considerado significativo $\mathrm{p}<0,05$.

\section{Resultados}

Participaram do estudo 54 profissionais de enfermagem, sendo 18 (33,33\%) enfermeiros e 36 (67,67\%) técnicos/auxiliares de enfermagem. A maioria dos profissionais $(53,70 \%)$ tinha até 39 anos; sexo feminino (81,48\%); casadas e/ou em união estável (62,96\%); com tempo de formação igual ou maior que 11 anos (68,52\%); tempo de trabalho no HE de até 5 anos (90,74\%); contratados pelo regime da Consolidação das Leis Trabalhista (CLT) (90,74\%); e graduadas (70,37\%).

A prevalência de alto e médio nível de estresse foi de 11,11\% e 46,30\%, respectivamente. Os profissionais com maiores proporções de estresse foram aqueles com idades até 39 anos (58,62\%), sexo masculino (60\%), solteiros (60\%), que afirmaram ter alguma religião (58,33\%), com menor tempo de formação $(76,47 \%)$, menor tempo de trabalho no $\mathrm{HE}(59,18 \%)$, os pós-graduados $(74,07 \%)$ e pertencentes à categoria profissional de enfermeiros (88,89\%). A análise mostrou associação significativa entre maiores níveis de estresse e as variáveis: menor tempo de formação ( $p$-valor=0,05), possuir pós-graduação (p-valor 0,01) e ser enfermeiro (p-valor=00). A regressão logística (OR-IC95\%) apontou aumento da probabilidade de maiores níveis de estresse em 113\% para enfermeiros, em 57\% para aqueles com menor tempo de formação e em $81 \%$ para aqueles com pós-graduação (Tabela 1).

Tabela 1 - Associação e Razão de chance de estresse ocupacional em profissionais de enfermagem que atuam na Unidade de Terapia Intensiva. Salvador, Bahia, Brasil $-2020 . \mathrm{N}=54$

\begin{tabular}{|c|c|c|c|c|}
\hline Variáveis & n Total & $\begin{array}{c}\text { Maiores níveis } \\
\text { de estresse } \\
\text { ocupacional (\%) }\end{array}$ & $\begin{array}{c}\text { Odds Ratio } \\
\text { (Intervalo de } \\
\text { Confiança 95\%) }\end{array}$ & P-valor \\
\hline \multicolumn{5}{|l|}{ Idade } \\
\hline Até 39 anos & 29 & 58,62 & $1,03(0,64-1,66)$ & 0,87 \\
\hline 40 ou mais & 23 & 56,52 & 1 & \\
\hline
\end{tabular}


Tabela 1 - Associação e Razão de chance de estresse ocupacional em profissionais de enfermagem que atuam na Unidade de Terapia Intensiva. Salvador, Bahia, Brasil - 2020. N=54 (conclusão)

\begin{tabular}{|c|c|c|c|c|}
\hline Variáveis & n Total & $\begin{array}{c}\text { Maiores níveis } \\
\text { de estresse } \\
\text { ocupacional (\%) }\end{array}$ & $\begin{array}{c}\text { Odds Ratio } \\
\text { (Intervalo de } \\
\text { Confiança 95\%) }\end{array}$ & P-valor \\
\hline \multicolumn{5}{|l|}{ Sexo } \\
\hline Homem & 10 & 60,00 & 1 & \\
\hline Mulher & 44 & 56,82 & $0,94(0,53-1,67)^{* * *}$ & 0,85 \\
\hline \multicolumn{5}{|l|}{ Raça } \\
\hline Não Negra & 7 & 57,14 & 1 & \\
\hline Negra & 47 & 57,45 & $1,00(0,50-1,99)^{* * *}$ & $0,98^{* * *}$ \\
\hline \multicolumn{5}{|l|}{ Estado Civil } \\
\hline Solteiro & 20 & 60,00 & 1 & \\
\hline Casado/União estável & 34 & 55,88 & $0,93(0,58-1,48)$ & 0,76 \\
\hline \multicolumn{5}{|l|}{ Religião } \\
\hline Sim & 48 & 58,33 & 1 & \\
\hline Não & 4 & 50,00 & $0,85(0,31-2,35)^{* *}$ & $0,74^{* *}$ \\
\hline \multicolumn{5}{|l|}{ Categoria Profissional } \\
\hline Enfermeira(o) & 18 & 88,89 & $2,13(1,40-3,24)$ & 0,00 \\
\hline $\begin{array}{l}\text { Auxiliar/Técnico de } \\
\text { enfermagem }\end{array}$ & 36 & 41,67 & 1 & \\
\hline \multicolumn{5}{|l|}{ Tempo de Formação } \\
\hline 1 a 10 anos & 17 & 76,47 & $1,57(1,02-2,40)$ & 0,05 \\
\hline 11 ou mais & 37 & 48,65 & 1 & \\
\hline \multicolumn{5}{|l|}{ Tempo de Trabalho no } \\
\hline \multicolumn{5}{|l|}{ Hospital de Ensino } \\
\hline 1 a 5 anos & 49 & 59,18 & 1 & \\
\hline 10 ou mais & 5 & 40,00 & $0,67(0,22-2,02)^{* * *}$ & $0,40^{* * *}$ \\
\hline \multicolumn{5}{|l|}{ Vínculo Empregatício } \\
\hline CLT & 49 & 57,14 & 1 & \\
\hline RJU & 5 & 60,00 & $1,05(0,49-2,23)^{* *}$ & $0,90^{* * *}$ \\
\hline \multicolumn{5}{|l|}{ Possui Graduação } \\
\hline Sim & 38 & 63,16 & $1,44(0,78-2,64)$ & 0,18 \\
\hline Não & 16 & 43,75 & 1 & \\
\hline \multicolumn{5}{|l|}{ Possui Pós-Graduação } \\
\hline Sim & 27 & 74,07 & $1,81(1,09-3,01)$ & 0,01 \\
\hline Não & 27 & 40,74 & 1 & \\
\hline
\end{tabular}

Fonte: Elaboração própria.

*Qui-quadrado; **Teste exato de Fisher.

As atividades relacionadas à assistência de enfermagem prestada aos pacientes consideradas como mais desgastante na UTI foram: enfrentar a morte do paciente $\mathrm{n}=31(57,41 \%)$ ( $\mathrm{p}$-valor $=0,01)$, orientar familiares dos pacientes críticos $n=26$ $(48,15 \%)$ (p-valor $=0,02)$, atender às emergências na unidade $\mathrm{n}=20(37,04 \%)$ ( $\mathrm{p}$-valor $=0,00)$, atender aos familiares dos pacientes críticos $n=20$ $(37,04 \%)$ ( $\mathrm{p}$-valor $=0,00)$, prestar os cuidados de enfermagem $\mathrm{n}=19(35,19 \%)$ ( $\mathrm{p}$-valor $=0,00)$, atender às necessidades dos familiares $n=18$ $(33,33 \%)$ ( $\mathrm{p}$-valor=0,00). O maior desgaste para desempenho de todas as atividades citadas esteve associado aos maiores níveis de estresse ocupacional, conforme Tabela 2.

A regressão logística (OR - IC95\%) revelou aumento da probabilidade de maiores níveis de estresse em 143\% para os profissionais que referiram maior desgaste durante a admissão do paciente, em 135\% para aqueles com maior desgaste durante o atendimento das emergências, em 135\% entre os profissionais com maior nível de desgaste no atendimento aos familiares dos pacientes críticos e em $113 \%$ para aqueles com maior desgaste no atendimento às necessidades dos familiares (Tabela 2). 
Tabela 2 - Associação e Razão de chance de estresse ocupacional relacionado à assistência prestada, em profissionais de enfermagem que atuam na Unidade de Terapia Intensiva. Salvador, Bahia, Brasil $-2020 . N=54$

(continua)

\begin{tabular}{|c|c|c|c|c|}
\hline Variáveis & n Total & $\begin{array}{c}\text { Maiores níveis } \\
\text { de estresse } \\
\text { ocupacional (\%) }\end{array}$ & $\begin{array}{c}\text { Odds Ratio } \\
\text { (Intervalo de } \\
\text { Confiança 95\%) }\end{array}$ & P-valor \\
\hline \multicolumn{5}{|c|}{ Admissão do paciente } \\
\hline $\operatorname{Sim}$ & 15 & 100,00 & $2,43(1,67-3,55)$ & 0,00 \\
\hline Não & 39 & 41,03 & 1 & \\
\hline \multicolumn{5}{|c|}{ Exame físico } \\
\hline Sim & 8 & 87,50 & $1,67(1,14-2,45)$ & 0,06 \\
\hline Não & 46 & 52,17 & 1 & \\
\hline \multicolumn{5}{|c|}{ Prescrição de enfermagem } \\
\hline $\operatorname{Sim}$ & 4 & 100,00 & $1,85(1,43-2,39)$ & 0,07 \\
\hline Não & 50 & 54,00 & 1 & \\
\hline \multicolumn{5}{|c|}{$\begin{array}{l}\text { Avaliar as condições do } \\
\text { paciente }\end{array}$} \\
\hline Sim & 9 & 88,89 & $1,73(1,20-2,51)$ & 0,03 \\
\hline Não & 45 & 51,11 & 1 & \\
\hline \multicolumn{5}{|c|}{$\begin{array}{l}\text { Atender as necessidades do } \\
\text { paciente }\end{array}$} \\
\hline $\operatorname{Sim}$ & 14 & 78,57 & $1,57(1,03-2,37)$ & 0,06 \\
\hline Não & 40 & 50,00 & 1 & \\
\hline \multicolumn{5}{|c|}{$\begin{array}{l}\text { Atender as necessidades dos } \\
\text { familiares }\end{array}$} \\
\hline Sim & 18 & 88,89 & $2,13(1,40-3,24)$ & 0,00 \\
\hline Não & 36 & 41,67 & 1 & \\
\hline \multicolumn{5}{|c|}{$\begin{array}{l}\text { Orientar o paciente para o } \\
\text { autocuidado }\end{array}$} \\
\hline Sim & 9 & 100,00 & $2,04(1,51-2,75)$ & 0,00 \\
\hline Não & 45 & 48,89 & 1 & \\
\hline \multicolumn{5}{|c|}{$\begin{array}{l}\text { Orientar os familiares para o } \\
\text { autocuidado do paciente }\end{array}$} \\
\hline Sim & 13 & 84,62 & $1,73(1,17-2,56)$ & 0,02 \\
\hline Não & 41 & 48,78 & 1 & \\
\hline \multicolumn{5}{|c|}{$\begin{array}{l}\text { Supervisionar o cuidado de } \\
\text { enfermagem prestado }\end{array}$} \\
\hline Sim & 11 & 90,91 & $1,86(1,30-2,66)$ & 0,01 \\
\hline Não & 43 & 48,84 & 1 & \\
\hline \multicolumn{5}{|c|}{ Orientar para a alta do paciente } \\
\hline Sim & 9 & 77,78 & $1,45(0,93-2,270$ & 0,17 \\
\hline Não & 45 & 53,33 & 1 & \\
\hline \multicolumn{5}{|c|}{$\begin{array}{l}\text { Prestar os cuidados de } \\
\text { enfermagem }\end{array}$} \\
\hline Sim & 19 & 84,21 & $1,96(1,27-3,01)$ & 0,00 \\
\hline Não & 35 & 42,86 & 1 & \\
\hline \multicolumn{5}{|c|}{$\begin{array}{l}\text { Atender as emergências na } \\
\text { unidade }\end{array}$} \\
\hline Sim & 20 & 90,00 & $2,35(1,49-3,69)$ & 0,00 \\
\hline Não & 34 & 38,24 & 1 & \\
\hline \multicolumn{5}{|c|}{$\begin{array}{l}\text { Atender aos familiares dos } \\
\text { pacientes críticos }\end{array}$} \\
\hline Sim & 20 & 90,00 & $2,35(1,49-3,69)$ & 0,00 \\
\hline Não & 34 & 38,24 & 1 & \\
\hline
\end{tabular}


Tabela 2 - Associação e Razão de chance de estresse ocupacional relacionado à assistência prestada, em profissionais de enfermagem que atuam na Unidade de Terapia Intensiva. Salvador, Bahia, Brasil

$-2020 . N=54$

(conclusão)

\begin{tabular}{l|c|c|c|c}
\hline Variáveis & n Total & $\begin{array}{c}\text { Maiores níveis } \\
\text { de estresse } \\
\text { ocupacional (\%) }\end{array}$ & $\begin{array}{c}\text { Odds Ratio } \\
\text { (Intervalo de } \\
\text { Confiança 95\%) }\end{array}$ & P-valor \\
\hline $\begin{array}{l}\text { Enfrentar a morte do paciente } \\
\text { Sim }\end{array}$ & 31 & 70,97 & $1,81(1,03-3,16)$ & 0,01 \\
$\quad$ Não & 23 & 39,13 & 1 & \\
$\begin{array}{l}\text { Orientar familiares dos } \\
\text { pacientes críticos }\end{array}$ & & & & \\
$\quad$ Sim & 26 & 73,08 & $1,70(1,04-2,77)$ & 0,02 \\
Não & 28 & 42,86 & 1 & \\
\hline
\end{tabular}

Fonte: Elaboração própria.

\section{Discussão}

Os resultados desta pesquisa evidenciaram, quanto à caracterização dos participantes, semelhança com outros estudos publicados no Brasil e em outros países, podendo contribuir para o conhecimento a respeito do estresse ocupacional da enfermagem em UTI. Encontrou-se predominância de profissionais do sexo feminino (81,48\%), com até 39 anos $(53,7 \%)$, casados ou em união estável (62,96\%), com tempo de formação superior a 11 anos (68,52\%), com a graduação como formação mínima (70,37\%). O tempo de trabalho predominante inferior a cinco anos e a forma de contrato pelo regime da Consolidação das Leis Trabalhista (CLT) é justificado pela recente adesão do hospital à Empresa Brasileira de Serviços Hospitalares, responsável pela administração da organização.

Estudo sobre estresse em profissionais de enfermagem em UTI realizado em São Paulo (SP) também identificou maior percentual de profissionais do sexo feminino $(79,2 \%)$ e solteiros $(50,9 \%)^{(14)}$. Na Espanha, estudo semelhante identificou, entre os participantes, 74,2\% de mulheres, com idade média de 42,9 anos e tempo médio de atuação na UTI de 11,35 $\operatorname{anos}^{(5)}$. No Sudão, pesquisa com a mesma temática reportou que a maioria dos participantes era do sexo feminino (79,1\%); na faixa etária entre 20 e 29 anos (76,3\%); solteira (77,7\%); e 50,7\% tinham até 3 anos de trabalho na UTI ${ }^{(8)}$. Na Mongólia, 95\% dos enfermeiros participantes de pesquisa sobre o mesmo assunto eram do sexo feminino ${ }^{(15)}$.
De acordo com o instrumento utilizado nesta pesquisa para a quantificação do estresse ${ }^{(13)}$, o profissional é classificado no nível médio de estresse com pontuação mínima de 120 pontos na EBS; já os que possuem de 239 a 357 pontos são classificados no nível alto. Os resultados da pesquisa evidenciaram que 57,4\% dos participantes estavam afetados pelo estresse ocupacional em nível médio ou alto. Esses números expõem a dimensão do problema no campo do estudo, uma vez que o estresse pode acarretar prejuízos à saúde física e emocional dos trabalhadores, além de ter repercussões na segurança e nos serviços de saúde, quando se prolonga o tempo de trabalho ${ }^{(5)}$. Do ponto de vista das características, o trabalho realizado na UTI impõe demandas físicas e mentais ${ }^{(9-10)}$. Por isso, é considerado um dos mais estressantes dentro do hospital ${ }^{(9,11)}$.

Os achados do estudo convergem com dados de pesquisa brasileira com 263 enfermeiros que trabalhavam em UTI, que identificou aproximadamente $60 \%$ de níveis médio a alto de estresse ${ }^{(16)}$. É também semelhante a outro estudo realizado entre profissionais de enfermagem que atuavam em UTI de hospitais de alta complexidade das capitais brasileiras, cujo resultado apontou nível médio de estresse em 60,1\% dos enfermeiros ${ }^{(17)}$. Outra pesquisa realizada na cidade São Paulo (SP) identificou que a maioria dos profissionais apresentava níveis médios de estresse $(77,4 \%)$ e $15,1 \%$ apresentavam níveis elevados ${ }^{(14)}$, portanto resultado mais expressivo do que foi identificado neste estudo. 
A relação entre o trabalho da enfermagem na UTI e o estresse também é relatada por outros autores no Brasil e em outros países ${ }^{(10-11,18)}$. No Sudão, pesquisa realizada em 14 hospitais públicos com enfermeiros intensivistas relatou nível elevado do estresse ocupacional ${ }^{(8)}$. Em outros países, como Irã ${ }^{(7)}$, Mongólia ${ }^{(15)}$, Espanha e Portugal $^{(19)}$, resultados que apontam níveis elevados de estresse também são reportados.

As maiores proporções de estresse foram identificadas entre profissionais com até 39 anos $(58,62 \%)$, do sexo masculino (60\%), solteiros (60\%), com alguma religião (58,33\%), com menor tempo de formação $(76,47 \%)$, menor tempo de trabalho no campo da pesquisa $(59,18 \%)$, mais anos de estudo $(74,07 \%)$ e entre os enfermeiros $(88,89 \%)$. O menor tempo de formação, ter cursado pós-graduação e ser enfermeiro foram associados positivamente ao estresse.

Ao se analisar tal resultado à luz da literatura corrente, encontra-se semelhanças e pontos de distanciamento. Quanto à relação entre o tempo de formado e o nível de estresse, resultados de pesquisa brasileira mostram dessemelhança com o encontrado neste estudo. As autoras mostram, em tal pesquisa, que profissionais com até cinco anos de formados apresentaram menores níveis de estresse; já os enfermeiros com 11 a 15 anos de formados apresentaram maiores níveis de estresse ${ }^{(17)}$.

Entretanto, a maior parte da literatura encontrada afirma que profissionais que trabalham na UTI por muito tempo apresentam níveis mais baixos de estresse ${ }^{(6)}$ em concordância com o encontrado nesta pesquisa. Enfermeiros no início da carreira podem apresentar níveis mais elevados de estresse quando comparados àqueles que já atuam há mais tempo na terapia intensiva. Assim, quanto maior o tempo de trabalho em UTI, menor é o estresse ocupacional. Tal segurança pode ser justificada pela maior habilidade demonstrada pelo profissional mais experiente frente às situações emergentes na terapia intensiva ${ }^{(6,18)}$. Resultados semelhantes também são relatados em estudos internacionais ${ }^{(8,19)}$.

Não se encontrou dados na literatura que apoiassem as diferenças nos maiores níveis de estresse entre os profissionais do sexo masculino.
A elevação do estresse entre os homens torna-se relevante, uma vez que a enfermagem é exercida predominante por mulheres e poder-se-ia esperar que os maiores níveis de estresse acompanhassem tal proporção. Uma das possíveis justificativas para esta diferença pode estar amparada na individualidade e subjetividade da percepção do estresse. A interação do sujeito com os agentes estressores de diversas ordens somada à capacidade individual de enfrentamento resulta no estresse ocupacional ${ }^{(20)}$, podendo supor-se que as mulheres, nesta pesquisa, apresentaram melhor adaptação aos agentes tensiogênicos.

Ser solteiro e não ter filho, assim como encontrado nesta pesquisa, também foi relacionado ao estresse em Portugal e na Espanha ${ }^{(19)}$. Esse resultado pode ser justificado pelo fato de que níveis mais elevados de estresse foram encontrados em profissionais mais jovens, os quais podem ainda não ter optado pela vivência de relação conjugal ou da paternidade/maternidade. Profissionais que relataram pertencer a alguma religião apresentaram maiores percentuais de estresse, embora não tenha sido encontrada diferença estatisticamente significante. Paradoxalmente, a adoção de uma religião e a prática da espiritualidade são descritas como estratégias de coping frente ao estresse laboral ${ }^{(10)}$. Pode-se supor que os níveis elevados de estresse encontrados $(57,4 \%)$ estariam acima da capacidade de enfrentamento dos profissionais, constituindo-se, assim, em risco para a ocorrência de doenças físicas e mentais.

Autores mostram não haver diferença significativa nos níveis de estresse quando é comparada a qualificação ${ }^{(8)}$. Essa informação diverge dos resultados desta pesquisa, que encontrou maiores índices de estresse entre profissionais pós-graduados, o que pode relacionar-se ao fato de os enfermeiros também possuírem mais anos de estudo do que os profissionais de nível técnico e apresentarem associação significativa com o estresse laboral na UTI. A associação entre o maior nível de estresse e o profissional enfermeiro pode guardar relação com o papel do enfermeiro nos serviços de saúde. Segundo a Lei do Exercício Profissional da Enfermagem no 
Brasil, o enfermeiro é responsável por planejar, organizar, coordenar e executar os serviços assistenciais da enfermagem de alta complexidade, o que demanda alto grau de responsabilidade e elevação da carga de trabalho ${ }^{(21)}$.

Dentre as atividades relacionadas à assistência de enfermagem prestada aos pacientes, conforme o domínio D da EBS $^{(13)}$, ações como o enfrentamento da morte do paciente $(57,41 \%$; $\mathrm{p}$-valor $=0,01)$, o atendimento às situações de emergência $(37,04 \%$; $\mathrm{p}$-valor $=0,00)$, a realização de cuidados de enfermagem junto aos pacientes gravemente enfermos $(35,19 \%$; p-valor $=0,00)$ e o desgaste durante a admissão do paciente foram relatadas como mais tensiogênicas.

O trabalho na UTI é descrito como um dos mais desgastantes para o profissional de enfermagem. A gravidade dos pacientes requer assistência qualificada e contínua, e o cuidado a eles dispensado é considerado complexo, demandando responsabilidade no tratamento de suporte à vida, além de estar acompanhado da sensação de impotência frente ao pior prognóstico ${ }^{(22)}$. Para o enfermeiro que atua na UTI, algumas habilidades são requeridas, como capacidade de liderança, transmissão de conhecimentos e controle emocional frente às situações-limite. Toda essa conjuntura concorre para maior desgaste físico e emocional ${ }^{(23)}$.

No que diz respeito à assistência ao paciente, o lidar com a morte e com as incertezas quanto ao tratamento são descritos como estressantes. Nesse enfrentamento, a dificuldade da enfermagem já vem sendo enfatizada em outras publicações ${ }^{(4,22)}$. Acredita-se que o ocupar-se com pacientes em situação de terminalidade possa desencadear nos profissionais sentimentos de angústia, impotência, perda, projeção da própria morte e frustração, pelo fato de não conseguir manter a vida. Diante disto, destaca-se a importância do estabelecimento de discussões sobre o tema desde a formação básica, para que o entendimento da morte como parte do curso de vida possa contribuir para o seu enfrentamento de forma natural ${ }^{(24)}$.

Outras atividades assistenciais identificadas neste estudo foram associadas a maiores níveis de estresse, como a orientação aos familiares de pacientes críticos ( $\mathrm{p}$-valor $=0,02)$ e o atendimento às necessidades dos familiares ( $\mathrm{p}$-valor $=0,00$ ), além do desgaste durante a admissão do paciente. Pesquisa de revisão da literatura aponta para relações tensiogênicas entre profissionais de saúde e familiares dos pacientes, citando como desmotivadoras e estressantes a falta de compreensão e de reconhecimento do trabalho da enfermagem pela família ${ }^{(23)}$. Podem-se citar também as dificuldades dos profissionais de enfermagem em lidar com o sofrimento e em atender as necessidades emocionais de pacientes e familiares ${ }^{(22)}$.

Este estudo identificou a probabilidade de aumento dos níveis de estresse (135\%) entre os profissionais com maior grau de desgaste no atendimento aos familiares dos pacientes críticos e em $113 \%$ para aqueles com maior desgaste no atendimento às necessidades dos familiares. Tal dificuldade da enfermagem em lidar com a família também pode decorrer de situações que permeiam o trabalho da enfermagem, como número reduzido de profissionais, desenvolvimento de grande número de atividades, falta de material, pouca sensibilidade e empenho para lidar com o tema, resistência às mudanças $\mathrm{e}$ apego às rotinas pré-estabelecidas. Assim, acredita-se que o estabelecimento de um processo comunicativo claro e o acolhimento humanizado do familiar na UTI possam contribuir para a superação de barreiras entre trabalhadores e familiares e para a minimização do estresse gerado nas relações com os familiares ${ }^{(25)}$.

Níveis elevados de estresse podem indicar que a equipe aproxima-se do desgaste, com possíveis prejuízos à vida pessoal e profissional ${ }^{(14)}$. Diante do que foi discutido, reafirma-se que profissionais de enfermagem que trabalham em terapia intensiva podem sentir-se, com mais frequência, rodeados por sensações que contribuem para o desenvolvimento do estresse ocupacional e de outras situações relacionadas, como cansaço mental, dificuldade de concentração, depressão, absenteísmo, ansiedade, alterações do humor e até mesmo a síndrome de Burnout. Sabe-se que essas manifestações podem afetar a qualidade de vida e repercutir negativamente na capacidade para prestar cuidados 
apropriados aos pacientes ${ }^{(8-10)}$, inclusive com a possibilidade de ocorrência de erros, retardo na recuperação e aumento de mortalidade ${ }^{(8)}$. No corpo físico, pode provocar diversas alterações no sistema cardiovascular, aparelho digestivo e musculoesquelético ${ }^{(24)}$.

Destaca-se a necessidade de o estresse ser gerenciado, no intuito de evitar o alcance de níveis elevados, com maiores prejuízos para o profissional, o serviço e a qualidade da assistência prestada $^{(20)}$. É importante, então, identificar suas causas e envidar esforços no intuito de amenizá-las, para que a equipe de enfermagem possa exercer seu trabalho de forma mais eficiente e as consequências relacionadas sejam reduzidas ao mínimo ${ }^{(7,22)}$.

Cita-se, como exemplo de estratégia de enfrentamento do estresse ocupacional na UTI, um estudo realizado no Irã, no qual um grupo de enfermeiros foi treinado para o desenvolvimento da resiliência. Os autores identificaram, após as intervenções, que houve queda nos níveis de estresse relacionado ao trabalho e aumento nos escores de resiliência, levando à conclusão de que a aplicação de estratégias de resiliência contribuem positivamente para o enfrentamento do estresse ocupacional em enfermeiros intensivistas ${ }^{(7)}$.

Esta pesquisa apresenta como limitação o fato de ter sido realizada com um número reduzido de profissionais e em apenas uma organização hospitalar. A coleta das informações em uma amostra ampliada e em mais de um hospital poderia agregar maior confiabilidade aos resultados obtidos. Pode-se afirmar que os achados contribuem para a ampliação do diálogo com a literatura corrente, uma vez que são reforçados por publicações anteriores e abrem espaço para a continuidade da exploração do tema por meio de pesquisas de intervenção. Contribui também para apontar o perfil dos profissionais de enfermagem mais vulneráveis ao estresse ocupacional que pode culminar com o adoecimento, dados importantes para implementação de estratégias preventivas.

\section{Conclusão}

Encontrou-se uma prevalência 57,4\% de estresse ocupacional em nível médio ou alto entre os profissionais de enfermagem que trabalhavam em terapia intensiva, com associação significativa relacionada ao menor tempo de formação, ter cursado pós-graduação e ser enfermeiro. Dentre as atividades inerentes à assistência de enfermagem em UTI, conforme o domínio D da EBS, identificou-se maior contribuição para o desenvolvimento de estresse em atividades como o enfrentamento da morte do paciente, a necessidade de atender e prestar orientações aos familiares dos pacientes críticos, o atendimento às situações de emergência e a prestação de cuidados de enfermagem a pacientes gravemente enfermos.

Os resultados sugerem as dificuldades da enfermagem em lidar com temas delicados e ainda escassamente abordados na formação básica, como o enfrentamento da morte e as relações com a família dos pacientes. Além disso, todo o contexto da assistência da terapia intensiva é permeado por fatores potencialmente estressores, os quais podem ser identificados e minimizados, quando possível. Tendo em vista que nem todas as questões são passíveis de modificação, sugere-se que estratégias individuais para o enfrentamento do estresse ocupacional em UTI possam ser identificadas, estimuladas e desenvolvidas. Resultados mais satisfatórios para a redução do estresse podem ser alcançados por meio da cooperação entre os níveis da gestão e os trabalhadores, com o desenvolvimento de espaço para escuta e construção compartilhada de estratégias para o apoio mútuo e o fortalecimento das relações na equipe.

Sugere-se que pesquisas de intervenção possam ser realizadas com o intuito de demonstrar a eficácia de técnicas e métodos que possam contribuir para a redução do estresse ocupacional na terapia intensiva. Um ambiente de trabalho menos estressante pode contribuir para um cuidado seguro e para o alcance de resultados melhores para o relacionamento intragrupo e deste com pacientes e familiares.

\section{Colaborações:}

1 - concepção, projeto, análise e interpretação dos dados: Rosana Santos Mota, Valdenir Almeida da Silva e Isadora Goncalves Brito; 
2 - redação do artigo e revisão crítica relevante do conteúdo intelectual: Rosana Santos Mota, Valdenir Almeida da Silva, Ângela de Souza Barros e Andreia Santos Mendes;

3 - aprovação final da versão a ser publicada: Rosana Santos Mota, Valdenir Almeida da Silva, Isadora Goncalves Brito, Ângela de Souza Barros, Olga Maria Brito dos Santos, Andreia Santos Mendes e Lorena de Carvalho Souza.

\section{Referências}

1. Hirschle ALT, Gondim SMG, Alberton GD, Rerreira ASM. Estresse e bem-estar no trabalho: o papel moderador da regulação emocional. Rev Psicol Organ Trab. 2019;19(1):532-40. DOI: http:// dx.doi.org/10.17652/rpot/2019.1.14774

2. Organización Mundial de la Salud. La organización del trabajo y el estrés. Ginebra; 2004 [cited 2020 Mar 20]. Available from: https://www.who.int/es/ emergencies/diseases/novel-coronavirus-2019

3. Silva C, Batista EC. Estresse ocupacional em enfermeiros e técnicos de enfermagem intensivistas de uma UTI adulto. $\mathrm{R}$ Interd [Internet]. 2017 jan/mar [cited 2019 Jan 21];10(1):118-28. Available from: https:// revistainterdisciplinar.uninovafapi.edu.br/index. $\mathrm{php} /$ revinter/article/view/1119

4. Geremias LM, Evangelista LF, Silva RC, Furtado DS, Silveira-Monteiro CA, Freitas CF. Prevalência do diabetes mellitus associado ao estresse ocupacional em trabalhadores bancários, Minas Gerais, Brasil. Rev Cuid [Internet]. 2017 [cited 2020 June 22];8(3):1863-74. Available from: http://www.scielo.org.co/pdf/cuid/v8n3/22160973-cuid-08-03-1863.pdf

5. Carrillo-García C, Ríos-Rísquez MI, Martínez-Hurtado R, Noguera-Villaescusa P. Nivel de estrés del personal de enfermería de la Unidad de Cuidados Intensivos de un hospital clínico universitário. Enferm Intensiva. 2016;27(3):89-95. DOI: http:// dx.doi.org/10.1016/j.enfi.2016.03.001

6. Campos BS, Oliveira EG, Nunes CR. O estresse entre os profissionais de enfermagem na Unidade de Terapia Intensiva. Rev Cient Interdiscip [Internet]. 2017 [cited 2020 Oct 22];1(2):1-7. Available from: https://recien.com.br/index.php/Recien/article/ view/298/pdf

7. Babanataj R, Mazdarani S, Hesamzadeh A, Gorji MH, Cherati JY. Resilience training: effects on occupational stress and resilience of critical care nurses. Int J Nurs Pract. 2019;25(1):e12697. DOI: https://doi.org/10.1111/ijn.12697

8. Mohamedkheir RA, Amara ZM, Balla SA, Mohamed HAA. Occupational Stress Among Nurses Working in Intensive Care Units in Public Hospitals of Khartoum State, Sudan 2016. Am J Health Res. 2016;4(6):166-71. DOI: 10.11648/j. ajhr.20160406.13

9. Souza AC, Faria CMA, Tavares JRS, Azevedo AS. Fatores que influenciam no desenvolvimento do estresse em enfermeiros de unidade de terapia intensiva. Rev Perspectivas Online: Biológicas Saúde [Internet]. 2018 [cited 2020 Oct 17];8(27 supl). Available from: https://ojs3. perspectivasonline.com.br/biologicas_e_saude/ article/view/1464/1162

10. Guida TSP, Nascimento AB. Fatores associados ao estresse e coping da equipe de enfermagem de UTI: uma revisão integrativa. Rev Enferm Atenção Saúde [Online]. 2019 ago/dez;8(2):150-66. DOI: https://doi.org/10.18554/reas.v8i2.3167

11. Moura RS, Saraiva FJC, Santos RM, Rocha KRSL, Barbosa VMS, Calles ACN, et al. Níveis de estresse da enfermagem nas Unidades de Terapia Intensiva. Rev enferm UFPE on line. 2019 mar;13(3):569-77. DOI: https://doi.org/10.5205/1981-8963-v13i03a 236549p569-577-2019

12. Khanade K, Sasangohar F, Sutherland SC, Alexander KE. Deriving Information Requirements for a Smart Nursing System for Intensive Care Units. Crit Care Nurs Q. 2018;41(1):29-37. DOI: 10.1097/CNQ.0000000000000183

13. Bianchi ERF. Escala Bianchi de Stress. Rev Esc Enferm USP. 2009 [cited 2020 Jan 21];43(Esp):1055-62. Available from: https://www. scielo.br/pdf/reeusp/v43nspe/a09v43ns.pdf

14. Padilha KG, Barbosa RL, Andolhe R, Oliveira EM, Ducci AJ, Bregalda RS, et al. Carga de trabalho de enfermagem, estresse/burnout, satisfação e incidentes em unidade de terapia intensiva de trauma. Texto Contexto-Enferm. 2017;26(3):e1720016. DOI: http://dx.doi.org/10.1590/0104-070720170 01720016

15. Bazarragchaa S, Purevdorj T, Turbat B, Enkhdalai D, Lkhagva B, Luvsan ME. Compassion fatigue among physicians and nurses working in the Intensive and Critical Care Units of Hospitals in Ulaanbaatar, Mongolia. Cent 
Asian J Med Sci. 2018;4(1):17-24. DOI: https://doi. org/10.24079/CAJMS.2018.03.003

16. Lopes Guerrer FJ, Ferraz Bianchi ER. Estresse dos enfermeiros atuantes em UTI nas regiões do Brasil. Enfermería Global. 2011 Abr;10(22). DOI: https://doi.org/10.6018/eglobal.10.2.121791

17. Lopes Guerrer FJ, Ferraz Bianchi ER. Caracterização do estresse nos enfermeiros de unidades de terapia intensiva. Rev Esc Enferm USP. 2008;42(2):355-62. DOI: http://dx.doi.org/10.1590/ S0080-62342008000200020

18. Ratochinski CMW, Powlowytsch PWM, Grzelczak MT, Souza WC, Mascarenhas LPG. O estresse em profissionais de enfermagem: uma revisão sistemática. Rev Bras Ci Saúde. 2016;20(4):341-6. DOI:10.4034/RBCS.2016.20.04.12

19. Baldonedo M, Mosteiro P, Queirós C, Borges E, Abreu M. Stress no trabalho em enfermeiros: estudo comparativo Espanha/Portugal. Int J Work Condit [Internet]. 2018 Jun [cited 2020 Apr 12];15:67-80. Available from: file:///C:/Users/Maria\%20Jos\%C3\%A9/ Downloads/2018_Stressnotrabalhoemenfermeiros_ estudocomparativoEspanha-Portugal_IJWC.15_ Baldonedo.et.al_p.67.80.pdf

20. International Labour Organization. Workplace stress: A collective challenge [Internet]. Geneva; 2016 [cited 2020 Mar 12]. Available from: https://www.ilo.org/wcmsp5/groups/public/--ed_protect/---protrav/---safework/documents/ publication/wcms_466547.pdf

21. Ribeiro AC, Rocha RPS, Rocha RAS. Fatores do estresse ocupacional na equipe de enfermagem: uma revisão integrativa. Connection line - rev Eletrôn Univag. 2018;19:98-105. DOI: 10.18312/ 1980-7341.n19.2018.1198

22. Brochado C, Ribas JLC. Estresse da equipe de enfermagem na UTI. Rev Saúde Desenvolv [Internet]. 2018 [cited 2020 Mar 10];12(13):44-57. Available from: https://www.uninter.com/ revistasaude/index.php/saudeDesenvolvimento/ article/viewFile/998/554

23. Teixeira LB, Veloso LUP, Ribeiro IAP, Oliveira TN, Cortez ACL. Estresse ocupacional na enfermagem atuante na unidade de terapia intensiva: uma revisão da literatura. Investig Enferm Imagen Desarr. 2017;19(2):195-211. DOI: http://dx.doi.org/10.11144/Javeriana.ie19-2.eoea

24. Silva VA, Menezes MR, Alves MB, Amaral JB, Souza AS. Enfermedad terminal de la persona mayor em hospitalización prolongada: relatos de enfermeras. Etica de los cuidados [Internet]. 2017 [cited 2019 Dez 10];10(19):1-6. Available from: http://www.index-f.com/eticuidado/n19/ et10586r.php

25. Poerschke SMB, Salbego C, Gomes IEM, Andrade A, Nietsche EA, Silva TC. Atuação da enfermagem frente aos sentimentos dos familiares de pacientes em Terapia Intensiva. Rev Fund Care Online. 2019;11(3):771-9. DOI: 10.9789/2175-5361.2019.v11i3.771-779

Recebido: 17 de setembro de 2020

Aprovado: 8 de novembro de 2020

Publicado: 19 de janeiro de 2021

A Revista Baiana de Enfermagem utiliza a Licença Creative Commons - Atribuição-NãoComercial 4.0 Internacional. https://creativecommons.org/licenses/by-nc/4.0/

Este artigo é de acesso aberto distribuído sob os termos da Licença Creative Commons (CC BY-NC). Esta licença permite que outros remixem, adaptem e criem a partir do seu trabalho para fins não comerciais. Embora os novos trabalhos tenham de lhe atribuir o devido crédito e não possam ser usados para fins comerciais, os usuários não têm de licenciar esses trabalhos derivados sob os mesmos termos. 